\title{
EGFR Mutated Lung Adenocarcinoma with Secondary Glaucoma as Early Manifestation: A Case Report
}

\author{
Ida Ayu Jasminarti Dwi Kusumawardani ${ }^{1 *}$, Venny Singgih ${ }^{1}$, Ni Wayan Candrawati1, Putu Yuliawati ${ }^{2}$, Herman \\ Saputra $^{3}$, I Gusti Ayu Sri Mahendra Dewi ${ }^{3}$ \\ ${ }^{1}$ Department of Pulmonology and Respiratory Medicine, Faculty of Medicine, Udayana University/Sanglah General \\ Hospital, Bali, Indonesia. \\ ${ }^{2}$ Department of Ophthalmology, Faculty of Medicine, Udayana University/Sanglah General Hospital, Bali, Indonesia. \\ ${ }^{3}$ Department of Pathology Anatomy, Faculty of Medicine, Udayana University/Sanglah General Hospital, Bali, \\ Indonesia.
}

\section{ARTICLE INFO}

\section{Article history:}

Received 17 December 2020

Received in revised form 22 January 2021

Accepted 25 January 2021

Available online 30 January 2021

\section{Keywords:}

Lung adenocarcinoma,

Neovascular glaucoma,

Orbital metastases.

\begin{abstract}
Background: Orbital metastases are rare in pulmonary adenocarcinoma and can be manifested as glaucoma.

Case: A 64-year-old male patient complained of swelling, redness, painful and protruded left eye. Physical and radiological examination revealed mass in the right lung, retrobulbar intraconal mass of the left oculi, and metastases in the ribs, liver, brain, and vertebrae. Result of bronchial mucosal biopsy showed adenocarcinoma. Epidermal growth factor receptor (EGFR) mutation examinations detected exon 19 deletions, therefore Gefitinib was given. Left orbital exenteration was performed and obtained a biopsy result of adenocarcinoma metastases.

Conclusion: Orbital metastasis occurs by hematogenous pathway due to direct access of systemic circulation to left orbital blood flow. Glaucoma as a symptom of orbital metastases can appear before primary tumor symptoms, because lung cancer often does not show specific symptoms until advanced stage. Definitive treatment consists of targeted therapy and surgical management for metastases.
\end{abstract}

\section{INTRODUCTION}

Adenocarcinoma is one of the subtypes of lung cancer with the highest incidence. ${ }^{1}$ Lung cancer, especially adenocarcinoma subtype metastasis to the ocular orbit, is a rare case. Metastasis to the orbit is obtained from $2-4.7 \%$ of cancer cases. ${ }^{2}$ Symptoms of metastatic lung cancer to the orbit include diplopia, proptosis, blurry vision, eye pain, ptosis, or retrobulbar mass. ${ }^{2}$ These metastatic symptoms can appear before symptoms in the primary tumor, because lung cancer often does not give specific symptoms until it reaches an advanced stage. ${ }^{3}$

Management of lung cancer with metastases to the eye includes chemotherapy, photocoagulation, cryosurgery, surgical resection, or radiotherapy. ${ }^{4}$ The development of targeted therapy in pulmonary adenocarcinoma with epidermal growth factor receptor (EGFR) mutations provides a therapeutic modality using tyrosine kinase inhibitors (TKI), which in some case reports provide better outcome to pulmonary adenocarcinoma with eye and orbital metastases. 5,6
A 64-year-old male patient complained of swelling, redness, painful and protruded left eye. Initially, the patient experienced a slowly-blurred lefteye and narrowed vision of the left eye, resulted in complete loss of vision accompanied by eye pain. He was diagnosed with left eye neovascular glaucoma accompanied by central retinal vein occlusion and retinal detachment. Medical therapy was given, and trabeculectomy surgery and intravitreal bevacizumab injection were planned after the intraocular pressure decreased. Later on, he complained of worsening right chest pain, coughing with white sputum accompanied byweight and appetite loss. The patient also complained about lower back pain that got worse over time, spreading to the right and left legs, accompanied by tingling, weakness, and numbness in both legs. The patient worked as a gold mine worker for 10 years, and worked as a driver for 20 years. History of smoking 1 pack of cigarettes per day for 30 years, and stopped 10 years ago. 


\section{CASE}

Physical examination revealed normal vital signs, physical examination of the left eye revealed proptosis, palpebral edema and hyperemia, conjunctival Chemosis, and corneal edema (Figure 1). Physical examination of the lungs revealed decreased vocal fremitus, dimming and decreased breath sounds in the right anterior thorax at the level of the fifth and sixth intercostal spaces. Abdominal examination revealed hepatomegaly and neurological examination revealed pathological reflexes in both legs.
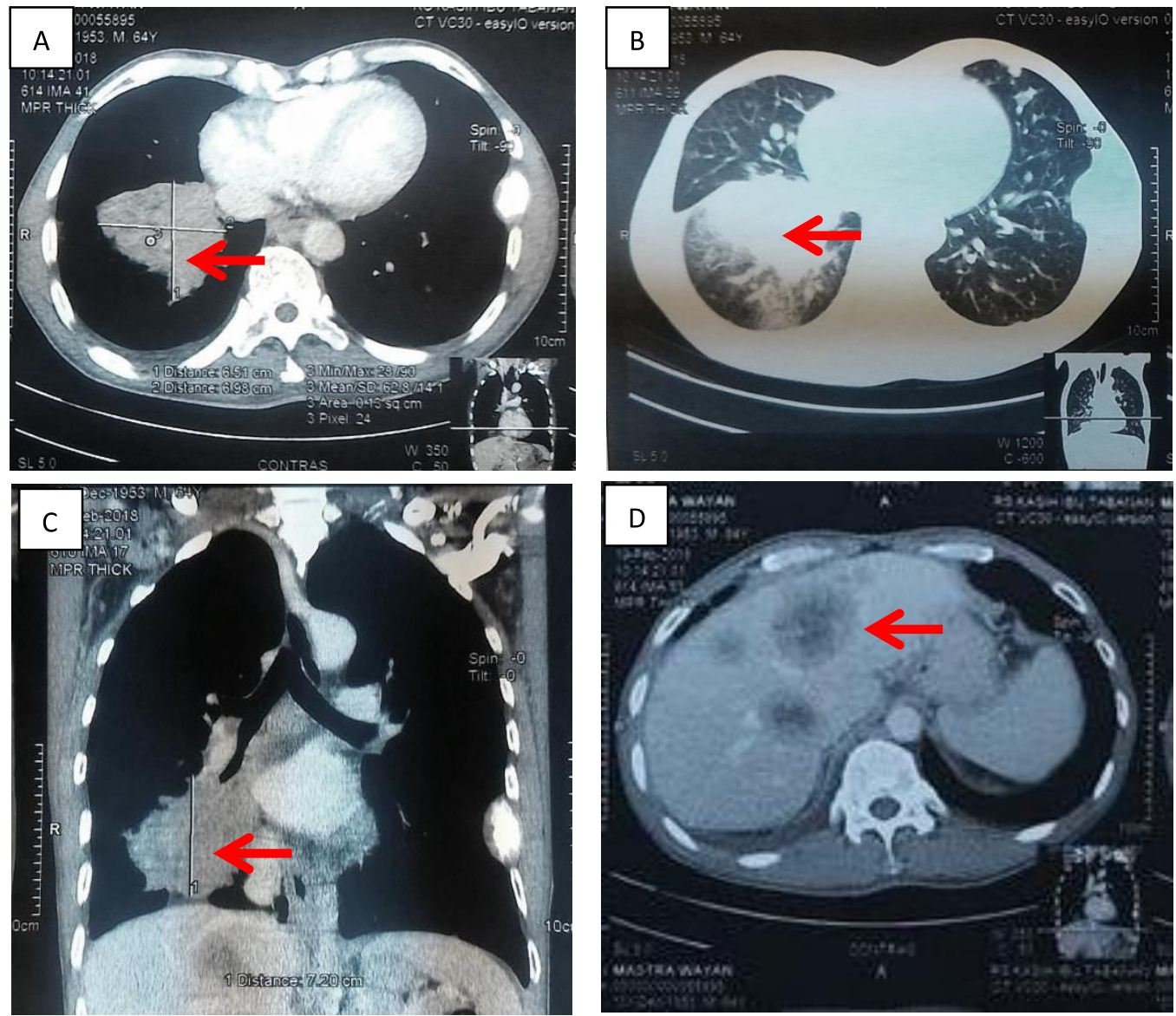
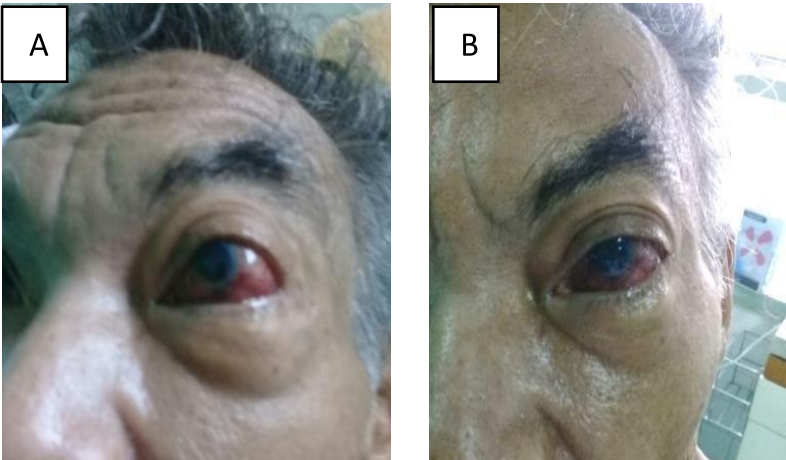

Figure 1. A) and B) Proptosis, palpebral edema and hyperemia, conjunctival Chemosis, and corneal edema of the left eye.

Figure 2. A), B), and C) Contrast-enhanced chest CT scan revealed a lobulated right subhilar tumor with pneumonic reaction (white arrows), multiple satellite nodules in both lungs, and destruction of the posterolateral 7th left rib D) Multiple nodules in the liver thatwere ring enhanced post contrast (black arrows).

Contrast-enhanced chest computed tomography (CT) scan revealed a lobulated right subhilar tumor with pneumonic reaction, and multiple satellite nodules inboth lungs. There were multiple nodules in the liver that were ring enhanced post contrast and destruction of the posterolateral $7^{\text {th }}$ left rib (Figure 2). 

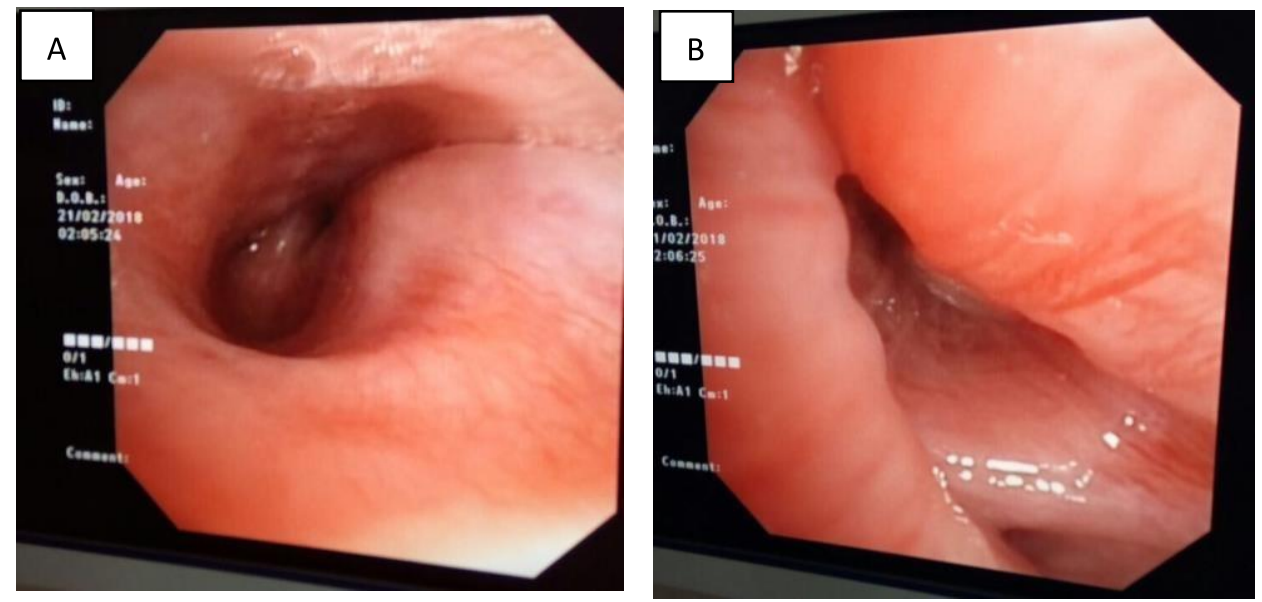

Figure 3. A) and B) Bronchoscopy revealed elliptical narrowing of the bronchi of the inferior lobe (except B6) due to extra-luminal pressing and infiltrative mucosa.

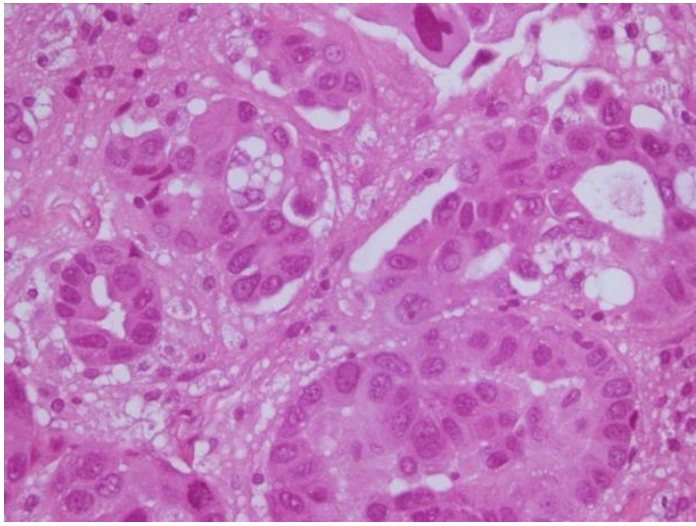

Figure 4. Bronchial mucosal biopsy showed proliferation of neoplastic cells which were infiltrative to the stroma. The neoplastic cells were round to oval with eosinophilic cytoplasm, arranged in acinar pattern. The neoplastic cells had round-tooval, moderately-to-severely pleomorphic nuclei withirregular nuclear membrane, high N/C ratio, and some with nucleoli. Histomorphological features were consistent with adenocarcinoma

Contrast-enhanced CT scan of the head and orbits showed retrobulbar intraconal mass of the left eye, which infiltrated the rectus and optic nerves, left maxillary sinus and caused proptosis; left eye retinaldetachment; multiple masses in the left occipital lobe, bilateral parietal lobe and left temporal lobe, suspicious of metastasis, cerebral edema with right midline shift; and soft tissue swelling of the left periorbital space (Figure 5).

Epidermal growth factor receptor (EGFR) examination detected deletion of exon 19, therefore tyrosine kinase inhibitor (TKI) was administered. The patient died 13 months later.
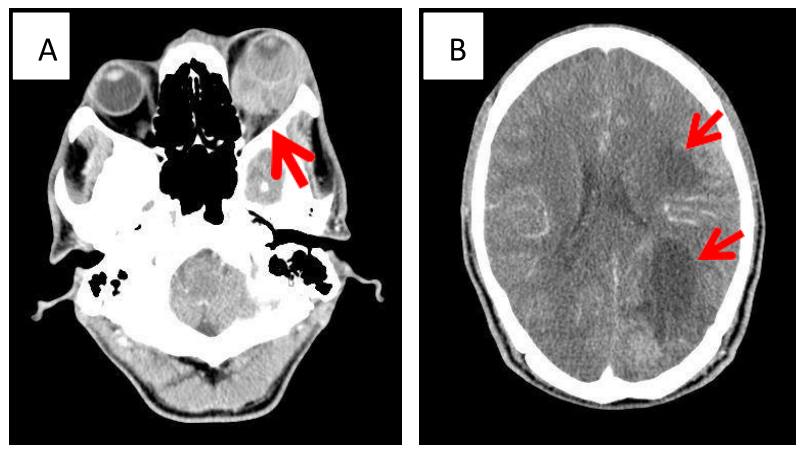

Figure 5. Contrast-enhanced CT scan of the head and orbits showed A) Retrobulbar mass of left eye, (black arrow). B) Multiple masses in the brain (white arrows)

\section{DISCUSSION}

Pulmonary adenocarcinoma is a type of non- small cell lung carcinoma that is quite common,approximately in $20-48 \%$ of cases of lung cancer. ${ }^{2}$ Adenocarcinoma is the most common subtype of lung cancer in women. Pulmonary adenocarcinoma is frequently found as an incidental finding $(20.6 \%)$. Symptoms that can appear are cough $(18.4 \%)$ and chest pain $(13.7 \%)$. Some of the patients experienced symptoms from their metastases $(8.7 \%)$. It is in contrast to other subtypes, which are generally preceded by complaints of respiration first. For example, squamous cell carcinoma, the most common symptoms that appeared at the beginning were cough (19.2\%) and coughing up blood (24\%). In this case, the patient had no previous respiratory symptoms, and the first symptom that arose was the symptom of orbital 
metastases, namely eye pain and visual disturbances. Respiratory symptoms that appeared first were coughing and chest pain.

Pulmonary carcinoma is often found in stage 4 with metastases. The most common metastatic pathways are lymphatic and hematogenic. Hematogenic metastases occur earlier with a high incidence of recurrence, thus reducing the patient's survival. Meanwhile, lymphatic metastases take longer. The most common metastatic sites for lung cancer are the brain, bones, and adrenal glands. Pulmonary adenocarcinoma, especially with EGFR mutations, often has metastases tothe brain. ${ }^{7}$

Adenocarcinoma is the lung cancer subtype with the most metastases, $43 \%$ of all lung cancer cases are with metastases. ${ }^{1}$ Lung cancer metastasis to the orbit is arare case. Orbital metastases account for $2-4.7 \%$ of cancer cases and is increasing from year to year. It is caused by improved cancer therapy that increases the median survival of cancer patients, thus providing opportunities for metastases to appear in new sites, developing diagnostic scanning tools, increasing use of fine needle biopsy, and the application of serological and molecular diagnostic techniques improve the detection of these lesions. In addition, the growing literature regarding orbital and ocular metastases raises awareness of these lesions. $^{2}$

Cancer metastasis to the orbit mostly found as breast cancer metastasis, but it can also be lung, prostate, thyroid, gastrointestinal tract, and kidney cancer metastases. ${ }^{5}$ Lung cancer has metastases in the eye in 0.2$6 \%$ of cases. ${ }^{8}$ Orbital metastasis generally occurs by hematogenous pathway. ${ }^{9}$ The most common orbital metastasis sites are highly vascularized areas of the uveal tract (choroid, iris, and ciliary body), especially the posterior segments of the eye, orbit, and optic nerve. ${ }^{3}$ Some studies indicate orbital metastases are more common in the left orbital, because the left common carotid artery is a direct branch of the aorta, thus the systemic circulation has direct access to the left orbital. However, other studies found no significant difference between the incidence of right and left orbital metastases. $^{2}$ Most of the ocular metastases are asymptomatic, and $30 \%$ of patients with orbital metastases have no previous history of primary tumor at the time metastases are detected. ${ }^{3}$
Symptoms of metastatic lung cancer to the orbit include diplopia, proptosis, decreased vision, eye pain, ptosis, or an orbital mass. ${ }^{3}$ Symptoms of metastasis can appear before symptoms in the primary tumor, because lung cancer often does not give specific symptoms until it reaches an advanced stage. ${ }^{3}$ The presence of orbital metastases causes an increase in intraocular pressure which later causes glaucoma. It is due to several mechanisms, including a growing or infiltrative mass effect that depresses the orbital structure. Progressive infiltration of the nerves exacerbates venous ischemia and results in neovascular glaucoma. ${ }^{10}$ The choroid mass causes bullous retinal detachment, and causes anterior displacement of the lens and iris. This gives rise to secondary angle closure glaucoma. Iris metastases cause secondary open-angle glaucoma due to tumor cells infiltration to trabecular meshwork that causes severe eye pain and blindness. ${ }^{5}$ In this case, the mass was located in posterior to ocular structure, infiltrative to the optic nerve, blood vessels, and infiltrative to the choroid layer and retina, causing retinal detachment.

Management of lung cancer with metastases in the eye includes chemotherapy, photocoagulation, cryosurgery, surgical resection, or radiotherapy. Radiotherapy may be given with low doses of external radiation therapy. In radioresistance cases, surgery is required. The orbital and ocular areas have different resistance to radiation. Effects that can arise from radiation are temporary or permanent loss of eyelashes, disruption of the lacrimal apparatus and lens. The walls of the sclera and cornea have high resistance toradiation. ${ }^{5}$ The development of targeted therapy, especially in pulmonary adenocarcinoma patients with EGFR mutations, provides a therapeutic modality using TKI, which in several case reports gave an improved response to pulmonary adenocarcinoma with eye and orbital metastases. ${ }^{5,6}$

Pulmonary adenocarcinoma patients with metastases and EGFR mutations who received TKI therapy had a 5-year survival rate of $14.6 \%$. Deletion of exon 19 and absence of extrathoracic and brain metastases provide better survival. ${ }^{11}$ The prognosis for ocular metastases is very low, with a life expectancy of 7.5 to 13 months for symptomatic patients, and 1.9 months for asymptomatic patients. 


\section{CONCLUSION}

Pulmonary adenocarcinoma is the subtype of lung cancer with the most distant metastases. Orbital metastases are rare. The initial manifestation that can appear is glaucoma. Orbital metastases, especially on the left side, can occur through hematogenous pathway due to the branching of the left common carotid artery which is a direct branching from the aorta, thus the systemic circulation has direct access to the blood flow in the left orbital and facilitates the nesting of tumor cells.

Management of lung cancer with metastases to the eye includes chemotherapy, photocoagulation, cryosurgery, surgical resection, or radiotherapy. In this case, EGFR mutations were evaluated and deletions of exon 19 were detected. Management in this case including administration of targeted therapy in the form of TKI.

\section{REFERENCES}

1. Riihimäki M, Hemminki A, Fallah $\mathrm{M}$, et al. Metastatic Sites and Survival in Lung Cancer. Lung Cancer 2014; 86: 78-84.

2. Ahmad SM, Esmaeli B. Metastatic Tumors of the Orbit and Ocular Adnexa. Curr Opin Ophthalmol 2007; 18: 405-413.

3. Sun L, Qi Y, Sun X, et al. Orbital Metastasis as the Initial Presentation of Lung Adenocarcinoma: A
Case Report. OncoTargets and Therapy 2016; 9: 2743-2748.

4. Lampaki S, Kioumis I, Pitsiou G, et al. Lung Cancer and Eye Metastases. Med Hypothesis, Discov Innov Ophthalmol J 2014; 3: 40-44.

5. Inoue M, Watanabe Y, Yamane S, et al. Choroidal Metastasis with Adenocarcinoma of the Lung Treated with Gefitinib. Eur J Ophthalmol 2010;20: 963-965.

6. Nair AG, Asnani HT, Mehta VC, et al. Tyrosine Kinase Inhibitors in the Treatment of Choroidal Metastases from Non-Small-Cell Lung Cancer: A Case Report and Review of Literature. Ocular Oncology and Pathology 2017; 3: 28-33.

7. Popper HH. Progression and Metastasis of Lung Cancer. Cancer Metastasis Rev 2016; 35: 75-91.

8. Macedo JE, Machado M, Araújo A, et al. Orbital Metastasis as a Rare Form of Clinical Presentation of Non-Small Cell Lung Cancer. J Thorac Oncol Off Publ Int Assoc Study Lung Cancer 2007; 2: 166167.

9. Shah SU, Mashayekhi A, Shields CL, et al. Uveal Metastasis from Lung Cancer: Clinical Features, Treatment, and Outcome in 194 Patients. Ophthalmology 2014; 121: 352-357.

10. Lin JJ, Cardarella S, Lydon CA, et al. Five-Year Survival in EGFR-Mutant Metastatic Lung Adenocarcinoma Treated with EGFR-TKIs. $J$ Thorac Oncol Off Publ Int Assoc Study Lung Cancer 2016; 11: 556-565.

11. Hoang A, Khine KT, Knight OJ, et al. A RareOcular Presentation of Metastatic Lung Cancer: Unilateral Anterior Chamber Angle, Bilateral Choroidal, and Multiple Intracranial Metastases. J Glaucoma 2017; 26: e93-e95. 